\title{
A Novel Data Clustering Algorithm Based on Gravity Center Methodology
}

\author{
Farag Hamed Kuwil ${ }^{a}$,*, Ümit Atila ${ }^{a}$, Radwan Abu-Issa ${ }^{\text {b }}$, Fionn Murtagh ${ }^{\mathrm{c}}$
}

\author{
${ }^{a}$ Department of Computer Engineering, Karabuk University, Karabuk, Turkey \\ ${ }^{b}$ Medical School, Ankara Yıldırım-Beyaztt-University, Ankara, Turkey \\ ${ }^{c}$ School of Computing and Engineering, Huddersfield University, Huddersfield, HDI 3DH, UK
}

\author{
A R T I C L EINFO \\ Keywords: \\ Algorithm \\ Cluster analysis \\ Euclidean distance \\ Gravity center \\ Partitional clustering
}

\begin{abstract}
A B S T R A C T
The concept of clustering is to separate clusters based on the similarity which is greater within cluster than among clusters. The similarity consists of two principles, namely, connectivity and cohesion. However, in partitional clustering, while some algorithms such as K-means and K-medians divides the dataset points according to the first principle (connectivity) based on centroid clusters without any regard to the second principle (cohesion), some others like K-medoids partially consider cohesion in addition to connectivity. This prevents to discover clusters with convex shape and results are affected negatively by outliers. In this paper a new Gravity Center Clustering (GCC) algorithm is proposed which depends on critical distance $(\lambda)$ to define threshold among clusters. The algorithm falls under partition clustering and is based on gravity center which is a point within cluster that verifies both the connectivity and cohesion in determining the similarity of each point in the dataset. Therefore, the proposed algorithm deals with any shape of data better than K-means, K-medians and K-medoids. Furthermore, GCC algorithm does not need any parameters beforehand to perform clustering but can help user improving the control over clustering results and deal with overlapping and outliers providing two coefficients and an indicator. In this study, 22 experiments are conducted using different types of synthetic, and real healthcare datasets. The results show that the proposed algorithm satisfies the concept of clustering and provides great flexibility to get the optimal solution especially since clustering is considered as an optimization problem.
\end{abstract}

\section{Introduction}

In the digitized world, with each passing day, people are producing information of enormous extent, and this information is then often stored as digital data ready for various analyses. Grouping of these data into certain categories is one of the most important types of analysis. Clustering is an unsupervised classification method for assigning data objects into set of disjoint groups called clusters so that points with high similarity drops in the same cluster (Jain, Murty, \& Flynn, 1999). Clustering is not only used for classifying data, it is also used for gaining insight into data by identifying distinctive features and detecting anomalies (Jain, 2010). Therefore, clustering can be considered as a key development area to observe unknown relationships between data points of existing sources and develop highly accurate and effective expert systems. Selecting an appropriate algorithm for clustering is a critical step since performances of the algorithms may vary according to the distribution nature of data and data encoding. In general, clustering algorithms can roughly be grouped into three categories such as hierarchical, density-based and partitional (Cheng-Fa Tsai, Han-Chang Wu, \& Chun-Wei Tsai, 2002; Verma et al., 2012).

Hierarchical clustering algorithms merge clusters with high similarity in its agglomerative stage or divide large clusters into smaller ones according to some required condition. These algorithms use tree structure to describe relationships among clusters. Hierarchical algorithms are used to find cluster hierarchy without the need to determine the number of clusters in advance and do not have a local minimum problem. Since these algorithms only consider local neighbors at each step, they do not incorporate any information regarding the global shape or size of the clusters in advance. Therefore, they are not always successful in separating overlapping clusters. Moreover, these algorithms are static so that a point previously assigned to a cluster cannot be moved to another cluster. They are also not suitable for large datasets since most of the hierarchical algorithms have quadratic or higher complexity in data size (Celebi, Kingravi, \& Vela, 2013; Cheng-Fa Tsai et al., 2002; Jain et al., 1999). Density-based algorithms utilize multiresolution grids and use dense grids to formalize clusters. The algorithms based on the grid density approach do not relate to data points but to the space value that covers the data points. These algorithms are fast in processing time regardless of the number of objects in the data set and can handle a large amount of noise in data sets, but not successful in high-dimensional data (Verma et al., 2012). Partitional algorithms, contrary to hierarchical algorithms, simultaneously find all clusters as a partition of the data and do not follow hierarchical structure (Jain et al., 1999). These algorithms can cover information regarding the global shape or size of the clusters by using appropriate distance metrics. There are two general principles in partitional algorithms. One is connectivity that concerns with affinity of data points with cluster centers and the other is cohesion that concerns with the extent of affinity among points themselves within the cluster. Partitional clustering in general has two types, one is crisp clustering and the other is fuzzy clustering. While each data point fits into only one cluster in crisp clustering, fuzzy clustering may put each data point into more than one cluster to some extent (Frigui \& Krishnapuram, 1999; Wang \& Rau, 2007). In case of overlapping, fuzzy clustering can deal with boundaries to solve this problem (Zhang \& Shen, 2014). Most of the partitional algorithms utilize optimization methods and iterative nature of those methods makes these algorithms sensitive to initialization and local minima. Difficulty in determining the number of clusters and their sensitivity to noise and outliers are the most critical drawbacks of these algorithms (Guha, Rastogi, \& Shim, 1998).

Drawbacks of the clustering algorithms mentioned here motivates us to

* Corresponding author.

E-mail address: kuwil73@gmail.com (F. H. Kuwil), umitatila@karabuk.edu.tr (U. Atila), ra691190@gmail.com (R. Abu-Issa), f.murtagh@hud.ac.uk (F. Murtagh). 
develop a more efficient clustering method and we propose a novel algorithm called Gravity Center Clustering (GCC) that takes in considering most of the limitations and drawbacks of most popular algorithms in clustering field. The proposed partitional algorithm performs clustering regardless of data distribution utilizing both connectivity and cohesion based on Euclidean distance and a threshold value calculated by Critical Distance Clustering Algorithm (Kuwil, Shaar, Topcu, \& Murtagh, 2019). GCC algorithm can successfully deal with outliers and noise in the dataset without the need for any parameters from user. In this paper we compare the results of GCC with other significant partitional clustering algorithms such as K-means, K-medoids and K-medians. The superiority on clustering quality of our proposed GCC algorithm is shown with experiments both on synthetic and real data taken from different web sources.

\section{Literature review}

Clustering is a well-known challenge and research on clustering for developing strong algorithms is still an ongoing endeavor. Determining number of clusters and discovering clusters with arbitrary shapes have been challenging problems in designing an effective clustering algorithm. All clustering algorithms have advantages and disadvantages and no single algorithm can be considered as perfect. While most of the current algorithms for clustering requires to specify number of clusters in advance, some others are not successful on dealing with outliers and high dimensional complex data.

In more than 50 years of clustering, the field brought many new ideas for getting effective and fast algorithms. Duda et al. (Duda, Hart, \& Stork, 2001) presented the general idea and references on clustering. Jain at al. and $\mathrm{Xu}$ and Wunsch (Jain, 2010; Xu \& Wunsch, 2005) presented comprehensive review studies addressing important issues on clustering. Some algorithms search for clusters as high-density regions separated by low density regions in the feature space. Ester et al. (Ester, Kriegel, Sander, \& $\mathrm{Xu}, 1996)$ proposed DBSCAN which is the most popular algorithm following this notion. Although density-based methods have native ability to deal with arbitrary shaped clusters, these methods have problems with high dimensional data because feature space is usually getting sparse and makes it difficult to distinguish high density regions from low density regions. Subspace clustering algorithms such as CLIQUE (Agrawal et al., 1998) solve this problem by detecting clusters embedded in lowdimensional subspace of the given high dimensional data. Zhou et al. (D. Zhou et al., 2004) presented a density-based partitioning and hierarchical algorithm named SUDEPICH. The algorithm first divides the data space into grids with equal sizes, sorts them by densities and then hierarchically merges those grids according to their density. In addition, some probabilistic models have been developed that use density function provided by a probabilistic mixture model (McLachlan \& Basford, 1988). To improve mixture models, some Bayesian models such as Latent Dirichlet Allocation (Blei, Ng, \& Jordan, 2003) and Pachinko Allocation (Li \& McCallum, 2006) model have been developed.

In hierarchical clustering the most well-known algorithms of this type can be viewed as single-link and complete-link. Day and Edelsbrunner (Day \& Edelsbrunner, 1984) presented characteristics of different agglomerative hierarchical clustering algorithms. Single-link algorithm starts with $\mathrm{n}$ number of clusters for $\mathrm{n}$ objects and then algorithm merges two closest clusters step by step into larger clusters until all the clusters are in a single cluster. Guha et al. (Guha et al., 1998) proposed an efficient hierarchical clustering algorithm for large datasets namely CURE that stops creating cluster hierarchy according to a given parameter $\mathrm{k}$ that indicates the number of clusters in a level of hierarchy. Their algorithm achieves good clustering results for arbitrary shaped clusters by utilizing multiple reference points to calculate distance between clusters. Gil-Garcia et al. (Gil-Garcia, Badia-Contelles, \& Pons-Porrata, 2006) proposed two hierarchical clustering algorithms namely Hierarchical Compact Algorithm and Hierarchical Start Algorithm based on maximum $\beta$-similarity graphs that produce clusters adjust well to arbitrary shapes. He et al. (He, Zhao, Zhang, Ramamohanarao, \& Pang, 2014) developed an effective clustering algorithm named Self-Increase Clustering (SIC) that depends on Euclidean distance(He et al., 2014)(He et al., 2014)(He et al., 2014)(He et al., 2014). The algorithm can identify clusters described as well-separated that can be either of convex or non-convex sets. SIC can be considered as special kind of agglomerative hierarchical clustering algorithm since it shares several common characteristics with them. In SIC algorithm, a new cluster is created that holds an arbitrarily selected object in each iteration. This cluster then gradually grows by merging with nearby clusters when certain conditions are met. A difference of SIC algorithm from other hierarchical clustering algorithms is that SIC stops creating clusters automatically and produces appropriate number of clusters. Unlike many other clustering algorithms, the SIC algorithm does not require the number of clusters to be created as an initial parameter. Rather, the SIC algorithm can automatically identify the number of clusters and isolate these clusters successfully according to the distribution of the input data set. The drawback of SIC is that it is not robust to outliers in the dataset. The minimal spanning tree (MST) based clustering algorithms are also hierarchical algorithms that extract clusters from a graph-based structure. These algorithms can detect clusters with irregular boundaries. MST-based clustering algorithms are convenient to use with high dimensional and large datasets. However, a quadratic time is required to construct an MST. Furthermore, the quality of clustering is reduced since outliers cause different densities in the clusters that makes the longest edges unreliable measure for separating the clusters (Halim \& Uzma, 2018). Murtagh and Contreras (Murtagh \& Contreras, 2016) developed a computationally effective clustering method for very large, very high dimensional and sparse data based on constructing random spanning paths. They used random projection to process high dimensional data which allows computationally effective hierarchical clustering using Baire metric.

Zhou et al. (R. Zhou, Shu, \& Su, 2015) proposed an MST based clustering algorithm called Adaptive Minimum Spanning Tree (AMST) to extract irregular shaped clusters. The general idea of the algorithm is first to determine the appropriate number of partitions in the region utilizing a validity index and then determining the significance of candidate clusters. Thus, algorithm finds the best part of the MST for clustering.

Another type of clustering is partitional clustering. K-means algorithm which is one of the most famous ones in clustering is part of partitional clustering. Despite its efficiency and simplicity, K-means algorithm has several drawbacks. Firstly, the algorithm may stick in local optimal solutions since it depends on cluster initialization. Therefore, the performance of the algorithm relies on initial cluster centers. Secondly, the number of clusters must be specified before starting clustering (Nanda \& Panda, 2014; Niknam \& Amiri, 2010; Zadeh, Fathian, \& Gholamian, 2014). This algorithm utilizes distance metrics to find similar data objects in the dataset which plays important role for the performance of clustering. Kmeans is based on Euclidean distance which gives best performance 
compared to other well-known distance metrics such as Manhattan, Chebychev and Minkowsky (Singh, Yadav, \& Rana, 2013). However, in connectivity-based K-means algorithm, tendency of Euclidean distance to generate hyper spherical clusters makes the algorithm inefficient for clustering data objects in real datasets with complex shape of clusters (Duda et al., 2001). Another partitional algorithm developed by Elfarra et al. (Elfarra, EL Khateeb, \& Ashour, 2013) uses concept of gravity. In this algorithm, each data point is assigned a gravity in the cluster and move toward the closest data point with higher gravity. As a result, each cluster has one point which is the centroid of that cluster. Drawback of this algorithm is the need for determining the number of clusters $\mathrm{K}$, the initial centroids and assigning each data point to one centroid before clustering. On the other hand, K-means algorithm has been modified in different ways. It is considered that good cluster initialization methods may increase the speed of the convergence of K-means. The study by Celebi et al. (Celebi et al., 2013) presents a review of many initialization methods but applying these methods may not be adequate especially when the number of clusters required is large. Fuzzy C-Means, a well-known extension of K-means developed by Dunn (Dunn, 1973) and later improved by Bezdek (Bezdek, 1981) uses a membership value to make a data point member of multiple clusters. Eschrich et al. (Eschrich, Jingwei Ke, Hall, \& Goldgof, 2003) proposed an algorithm called brFCM that speeds up K-means or Fuzzy CMeans without adversely affecting partition quality by reducing the number of different patterns that need to be clustered. Kaufman and Rousseeuw (Kaufman \& Rousseeuw, 1990) proposed K-Medoid which uses median of the data to represent clusters instead of the mean. Determining the number of clusters automatically has also been studied extensively. Most of the proposed algorithms for this purpose are run with different number of clusters $\mathrm{K}$ and then the best value of $\mathrm{K}$ is chosen based on predefined criterion. Minimum Message Length (MML) was used by Figueiredo and Jain (Figueiredo \& Jain, 2002) to estimate K utilizing Gaussian Mixture Model (GMM). Pelleg and Moore (Pelleg \& Moore, 2000) developed an algorithm called X-Means that automatically determines the best value of $\mathrm{K}$ by optimizing Bayesian Information Criterion (BIC) or Akaike criteria which are used to score each model with different number of clusters $\mathrm{K}$. The drawback of X-Means is the difficulty in determining the number of different $\mathrm{K}$ values to compare, because when the size of dataset is very large, range of possible number of clusters gets large as well. Another proposed algorithm is G-Means (Hamerly \& Elkan, 2003) to estimate the best value of $\mathrm{K}$. The algorithm starts with a small value of $\mathrm{K}$ and applies statistical normality test for each cluster. If test results with high confidence of Gaussian Distribution, the cluster is divided into two clusters and the test continues for the rest of the clusters. However, for determining the appropriate number of clusters these algorithms need to perform clustering process first. In addition, clustering methods that utilize meta-heuristic optimization algorithms are worthy to be reviewed here too. Meta-heuristic optimization algorithms such as Artificial Bee Colony (Karaboga \& Ozturk, 2011), Ant Colony Optimization (Shelokar, Jayaraman, \& Kulkarni, 2004) and Tabu Search (Al-Sultan, 1995) were utilized to decrease the risk of being trapped in local optima which is one of the main drawbacks of Kmeans. In another study, Das et al. (Das, Abraham, \& Konar, 2008) proposed a clustering algorithm suitable for inseparable and large datasets based on Multi-Elitist Particle Swarm Optimization (PSO) which uses similarity measure instead of Euclidean distance. Ahmadyfard and Modares (Ahmadyfard \& Modares, 2008) proposed a hybrid algorithm that uses Kmeans and PSO to solve the problems of K-means. Another study presented by Saida et al. (Saida, Kamel, \& Omar, 2014) discussed a hybrid clustering algorithm based on Cuckoo Search (CS) method and K-means. CS method is robust, efficient and easy to implement for many types of optimization issues. However, this algorithm needs to specify some internal parameters such as worst nest probability. Main drawback of using meta-heuristic optimization algorithms for clustering is the need to specify extra parameters to achieve best performance.

\section{The proposed algorithm}

Generally, data clustering concept is based on the similarity which consists of two principles, namely, connectivity and cohesion, where connectivity concentrates on the relation of points with the cluster centers while cohesion concentrates on the relation among points themselves within the cluster. The proposed Gravity Center Clustering (GCC) algorithm is similar to $\mathrm{K}$-means as both fall under partition clustering and depend on the centroid. In K-means algorithm, the centroid is called cluster center and is selected randomly then updated by iteration, while in GCC algorithm, it is called gravity center "GC" and it is a point which is also within cluster chosen based on lambda $(\lambda)$ from CDC algorithm (Kuwil et al., 2019) that represents a threshold. Another important difference between the two algorithms is that, while in K-means, the main idea is to divide the dataset points just according to connectivity, where the points are assigned to a cluster based on the distance between them and the centroid of the cluster, GCC algorithm considers both principles connectivity and cohesion. These principles are used to determine the affiliation of each point in the dataset. As a result, while K-means is unable to discover clusters that do not resemble the circular shapes, GCC can deal with any shape of data. In GCC algorithm, $\lambda$ is used to determine the threshold and identify the required similarity inside clusters using Euclidean distance. Moreover, there is no need to specify any parameters beforehand to implement the algorithm, but two coefficients $\lambda$ and $n$ and an indicator sigma $\sigma$. These coefficients can be used especially in non-ideal cases to provide flexibility and comprehensiveness, where $n$ represents the minimum number of points in each cluster and $\lambda$ represents the threshold used to increase or decrease the number of clusters. Thus, $\lambda$ and $n$ can be changed from default values to desired values in case of challenges such as the presence of outliers, overlapping or weak clusters which consist of insufficient number of points to recognize a pattern.

\subsection{Steps of GCC algorithm}

Generally, the GCC algorithm depends on searching for the smallest cluster in terms of number of points and then put aside and search for the next one from the dataset. This process is repeated until all the data points are assigned to an appropriate cluster. The basic idea is to find the critical distance $\lambda$, which is used to determine a cluster with minimum number of effective points that share the same threshold. Then, a point that has lowest edge value is selected as gravity center for this cluster. The symbols and abbreviations which are used in the algorithm are defined as in Table 1.

Table 1.

The symbols and abbreviations used in the algorithm. 


\begin{tabular}{ll}
\hline Exp_No & Vertical axis \\
\hline D_M & Distance matrix \\
P2PS_M & Peer to peer similarity matrix \\
$\lambda$ & Critical distance \\
k & Number of clusters \\
S_DM_V & Sorted Distance Matrix Value \\
S_DM_P & Sorted Distance Matrix Position \\
T_E_M & Threshold Edge Matrix \\
A_S_M & Active Set Matrix \\
$C_{(k)}$ & Cluster k \\
$G C_{(k)}$ & Gravity Center for cluster k. \\
N & Number of object (rows in dataset) \\
\hline
\end{tabular}

Assume that the dataset points each representing arbitrary objects are given as $p_{1}, \ldots, p_{n}$ and their similarities are $d_{i j}=s\left(p_{i}, p_{j}\right)$ measured according to Euclidean distance as shown in Eq. (1).

$d_{i j}=\sqrt{\left(x_{p i}-x_{p j}\right)^{2}+\left(y_{p i}-y_{p j}\right)^{2}}$

Detailed steps of the algorithm are as follows:

1- Distance matrix D_M $\in \mathbb{R}^{\mathrm{n} \times \mathrm{n}}$ is formed defined by $D_{-} M_{i, j}=d_{i j}$ if $i \neq j$ and $D_{-} M_{i, i}=0$. Order of the points in D_M has the same order as in the original dataset. Thus, the row number represents the position of a point.

2- For each point $p_{i}$, find $p_{j}$ that makes $d_{i j}$ minimum (highest similarity) and form Peer-to-Peer similarity matrix P2PS_M $\in \mathbb{R}^{\mathrm{n} \times 3}$ defined by P2PS_M $=\left[p_{i}, p_{j}, d_{i j}\right]$. Then, critical distance is determined as $\lambda=$ $\max \left(d_{i j}\right)$ in matrix P2PS_M.

3- Two matrices named S_DM_V $\in \mathbb{R}^{\mathrm{n} \times \mathrm{n}}$ and $\mathrm{S}_{-} D M_{-} \mathrm{P} \in \mathbb{R}^{\mathrm{n} \times \mathrm{n}}$ are created. First, every row in D_M is put in ascending order and while distance values are stored in S_DM_V original column numbers of points (positions) are stacked in S_DM_P.

4- Threshold and Edge Matrix T_E_M $\in \mathbb{R}^{\mathrm{n} \times n-1}$ is constructed from S_DM_V matrix. Let ordered distances in a row of S_DM_V be defined as $d_{1}, \ldots, d_{n}$ then each row of T_E_M is defined by T_E_M $=\left[d_{k}-d_{k-1}\right]$ where $k=2, . ., n$. First value in each row of T_E_M which is bigger than $\lambda$ represents the separation case for nearest cluster where $p_{k}$ is named as threshold representing the first point of the next cluster and $p_{k-1}$ is named as edge representing the last point in the current cluster.

5- A set of objects with a common threshold is selected which is called the active set in current cluster and stored in the Active Set Matrix A_S_M $\in$ $\mathbb{R}^{\mathrm{k} \times 3}$ where $\mathrm{k}$ is a varying number that denotes the number of points in the active set. Separation cases in all rows of T_E_M are found and values in the same column are determined. Then, for each value sharing the same column in T_E_M, an edge point distance denoted by $d_{k-1}$ is selected from S_DM_V matrix and added to A_S_M. Other two columns added to A_S_M are corresponding positions of threshold points in S_DM_P matrix denoted by $p_{k}$ and original positions of points in the dataset (row number).

6- The minimum edge value in matrix A_S_M represents the gravity center of current cluster and denoted by $G C_{(k)}$ if the current cluster is $\mathrm{k}$. The row number of minimum edge (gravity center) value in A_S_M is selected in matrix S_DM_P and all the points until encountering the position of minimum edge value are put in current cluster $\mathrm{k}$.
7- Excluding those points assigned to cluster k, a new D_M is constructed for being used to create next clusters.

8- Steps between 2 and 7 is repeated until $\mathrm{N}=0$, so that all dataset points are distinctive in different clusters.

Steps of GCC algorithm can be summarized as follows.

Input: Distance matrix $\mathrm{D}_{-} \mathrm{M} \in \mathbb{R}^{\mathrm{n} \times \mathrm{n}}$ and P2PS_M $\in \mathbb{R}^{\mathrm{n} \times 3}$

- Find maximum distance in P2PS_M which represents $\lambda$

- From matrix D_M compute distance values and store in S_DM_V $\in \mathbb{R}^{\mathrm{n} \times \mathrm{n}}$ and their positions in S_DM_P $\in \mathbb{R}^{\mathrm{n} \times \mathrm{n}}$

- From matrix S_DM_V construct T_E_M $\in \mathbb{R}^{\mathrm{n} \times \mathrm{n}-1}$

- From matrix T_E_M construct A_S_M $\in \mathbb{R}^{\mathrm{k} \times 3}$

- Find minimum threshold value in A_S_M for cluster $\mathrm{k}$ denoted by $G C_{(k)}$

- Select the row in S_DM_P indicated by $G C_{(k)}$

- Select all the points starting from the first column of S_DM_P until encountering the position value of $G C_{(k)}$ and put the points in current cluster $\mathrm{k}$.

- Repeat previous steps until all dataset points are distinctive in different clusters

Output: Clusters $\mathrm{C}_{1}, \ldots, \mathrm{C}_{\mathrm{k}}$

\subsection{Graphical Representation of GCC}

In order to facilitate the understanding of how algorithm works, it is explained through a scenario illustrated in Fig. 1. Assume two points are given as $p_{1}$ and $p_{2}$ where $p_{1}$ is the last point in $C_{1}$ (edge) and $p_{2}$ is the first point in $C_{2}$. Gravity center is indicated by GC and active set consists of points which share the same threshold with GC. What is required is the determination of gravity center of $C_{1}$, as well as the threshold that separates it from $C_{2}$.

Let the distances be arranged in ascending order between the GC and all the points and defined as $d_{1}, \ldots, d_{n}$. Each distance is subtracted from the distance of next point and compared to $\lambda$. According to Figure $1, d_{2}-d_{1}>$ $\lambda$ means $p_{2}$ is a threshold and $p_{1}$ is an edge for $C_{1}$. The gravity center is chosen from active set which has minimum edge value and all the points denoted by $p_{i}$ which have distances to GC not satisfying the condition $d_{i}-$ $d_{i-1}>\lambda$ are assigned in $C_{1}$.

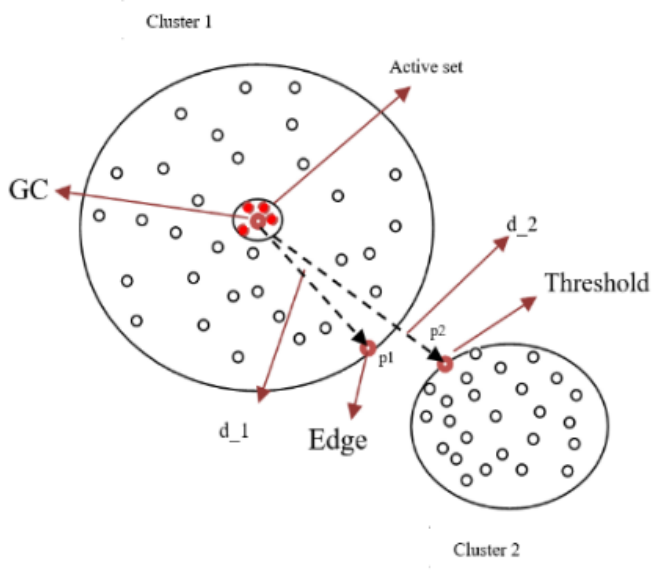

Fig. 1. A graphical illustration of GCC

\subsection{Discussion of the algorithm}


GCC algorithm provides the optimal number of clusters that are verified to be the general concept, however, most nature of real data distribution is random, and this led getting ideal clusters complicated. The number of clusters is not required a priori by the GCC algorithm because clusters are distinguished one after the other sequentially until their completion. The idea of the GCC algorithm is to verify both connectivity and cohesion principles for every cluster. The connectivity selects the least distances between GC and the rest of cluster points, while the cohesion can be represented by the differences of distances of two consecutive points to GC. GCC algorithm includes two coefficients and an indicator that makes it possible to overcome the problem of dataset distribution.

\subsubsection{Coefficients of $\lambda$ and $n$}

$\lambda$ is the first coefficient which depends on similarity criteria. Value of $\lambda$ is the largest distance which is also the weakest similarity that connect points within the cluster. Thus, once $\lambda$ is obtained the points inside the cluster can be connected and on the other hand obtaining the threshold value (the first value bigger than $\lambda$ ) lets to separate clusters from each other. In fact, $\lambda$ is used as a threshold when the data is completely subject to clustering. Otherwise, an alternative value of $\lambda$ is required to obtain acceptable results whenever possible as it will be illustrated in third group of experiments. The second coefficient $\mathrm{n}$ depends on quorum criteria through determining the minimum number of points in each cluster whenever necessary and quorum $=\mathrm{n}+1$ because it deals with A_S_M which comes from T_E_M. To do so, there are two conditions of the composition of the cluster. The distances to connect these points together must be less than or equal to $\lambda$ and the distance between this cluster and the nearest one must be bigger than $\lambda$. However, if the number of points in this cluster does not reach the quorum (n) that specified by the user, it will be combined with the closest cluster after the completion of the composition of all the clusters. It is noticeable that this coefficient does not merge similar clusters which are close to each other, but it can merge according to cluster size, since a small cluster size is a clear indication of weak clustering which make it difficult to extract any pattern.

\subsubsection{Sigma ( $\sigma)$ indicator}

When $\lambda$ is large enough to include all the dataset points in one cluster it has to be substituted by an alternative value that allows to create clusters. Thus, a value which plays the role of acceptable outlier named Upper Limit Outlier (ULO) or its multiplies need to be defined. In GCC, the first value bigger than ULO is considered as the threshold where the extremism begins. ULO is formulated with the following Eq. (2).

$$
U L O=Q_{3}+1.5(I Q R)
$$

Here, $Q_{3}$ is the interquartile range of the 95 th percentile and IQR is the interquartile range division that can be calculated using Eq. (3) given below where $Q_{1}$ is the interquartile range of the 5 th percentile.

$$
I Q R=\left(Q_{3}-Q_{1}\right) / 2
$$

According to CDC algorithm (Kuwil et al., 2019), the distances used within clusters are located in a matrix P2PS_M. ONE-WAY ANOVA analysis is used to identify ULO, since $\lambda$ is affected only by the extreme outliers. Therefore, 95th/5th percentile is used instead of 75 th $/ 25$ th percentile for specifying ULO value, which make it closer to the alternative value of $\lambda$. By knowing ULO values, it is possible to determine whether the $\lambda$ is suitable to represent the threshold of each cluster where a new indicator is calculated as $\sigma=\mathrm{ULO} / \lambda$. This indicator is used to select $\lambda$ and when $\sigma$ is not close to zero, it means $\lambda$ is sufficient to form clusters and the distances within each cluster are homogeneous. Whenever $\sigma$ value is close to zero, it indicates the need of using multiples of ULO as an alternative value of $\lambda$.

\subsubsection{Computational complexity of GCC}

Complexity of the algorithm depends on the overall time it requires to partition the dataset into clusters. Time to obtain clusters directly depends on the number of data points. The GCC algorithm consists of four main stages. The first stage is to obtain the P2PS_M by calculating the nearest neighbor for the whole dataset points. Thus, required time for this stage is $\mathrm{O}(\mathrm{n})$. In the second stage, we need to put distances of D_M in ascending order with their position for every row and this requires $\mathrm{O}\left(\mathrm{n}^{2}\right)$ time and then we find the differences between every adjacent points for all dataset and this is completed in $\mathrm{O}\left(\mathrm{n}^{2}\right)$ time. In the third stage we find the first threshold for every point in $\mathrm{O}(\mathrm{n})$ time. Finally, last stage selects gravity center from the active set for every cluster in $\mathrm{O}(\mathrm{n} \log n)$ time and then separates clusters also in $\mathrm{O}(\mathrm{n} \operatorname{logn})$ time. Consequently, in the worst case, the total time required for $\mathrm{GCC}$ is $\mathrm{O}(\mathrm{n})+\mathrm{O}\left(\mathrm{n}^{2}\right)+\mathrm{O}\left(\mathrm{n}^{2}\right)+\mathrm{O}(\mathrm{n})+\mathrm{O}(\mathrm{n} \log n)+\mathrm{O}(\mathrm{n} \log n)=$ $\mathrm{O}\left(\mathrm{n}^{2}\right)$.

\section{Experimental analysis and the results}

The proposed algorithm was implemented using MATLAB. The experiments were conducted under three groups with different synthetic and real data to demonstrate the characteristics of the algorithm. The first group addresses an optimal case where the dataset has full compatibility with the concept of clustering and default value of $\lambda$ and $n$ are used for the algorithm, while the second group demonstrates how to reduce the number of clusters by merging weak clusters to the most similar ones by using coefficient $n$. Finally, the third group of experiments illustrates the use of both coefficients $\lambda$ and $n$ to deal with outliers and overlapping. In all resulting figures, each cluster that contains a point in black color represents the GC for the cluster.

Partitional type clustering algorithms are considered in two groups as hard and soft clustering algorithms. Since our proposed algorithm is hard partitional clustering algorithm, in the first group of experiments all the results of GCC were compared with other well-known hard partitional type clustering algorithms such as K-means, K-medoids (Danyang Cao \& Bingru Yang, 2010) and K-medians (Kauffmann L., 1987). Unlike Kmeans, K-medoids chooses data points as cluster centers and performs clustering by minimizing the sum of the dissimilarity between each point and its cluster center. Compared to $\mathrm{K}$-means, $\mathrm{K}$-medoids is not sensitive to outliers but has larger calculation. On the other hand, K-medians algorithm uses Manhattan distance also known as 1-norm distance instead of Euclidean distance and cluster center minimizes the 1-norm distance to all points within that cluster which is also median of the cluster. Using medians instead of means of the points makes K-medians more robust to outliers.

\subsection{Experiments group 1: Comparing GCC with K-means, K-medoids and $K$-medians using synthetic data}


Eight experiments were performed using GCC algorithm in which the result is constant and does not change on each implementation because it does not depend on any initial random values. Table 2 shows the default coefficients used for GCC in the first group of experiments. The values of threshold are equal to $\lambda$ in all experiments in which the experiments were entirely consistent with the concept of clustering where all results show that no outliers or overlapping exist. When the value of the second coefficient was 2 as a default value, meaning that the minimum number of points in each cluster is 3 , there are no weak clusters that are required to merge. Note that $\sigma$ is not close to zero, therefore, $\lambda$ is suitable to form clusters in all experiments in this group.

Table 2.

The coefficients of the first group of experiments.

The results of the first group of experiments are presented in Fig. 2 where the first column shows results of GCC and others present the results of K-means, K-medoids and K-medians respectively. Since K-means, Kmedoids and $\mathrm{K}$-medians algorithms strongly depend on initial random centers, each independent run of these algorithms mostly produces different results. As seen in Fig. 2, GCC algorithm successfully gives results that is compatible with the concept of clustering and thus, results of GCC can be taken as reference for true clustering in order to understand how other algorithms perform. Also, it should be noted for Fig. 2 that, when the number of clusters is more than the number of main colors used in MATLAB, different clusters may be shown in same color. For example, in experiment 2, there are two different clusters shown in red color. In experiment 1 , all algorithms except $\mathrm{K}$-medians give true clustering results. Here, K-medians algorithm fails clustering because it cannot separate data showed with red color and puts both group of points in one cluster. At the same time, it separates data into two clusters as shown with cyan and magenta colors which should be put in one cluster. In experiments 2, 5 and 8, all algorithms except GCC give incorrect clusters and fail. As seen in

\begin{tabular}{clllcl}
\hline Exp. No. & ULO & $\lambda$ & $\sigma$ & Threshold & $\mathrm{N}$ \\
\hline 1 & 2.397 & 2.000 & 1.198 & 2.000 & 2 \\
2 & 2.390 & 3.836 & 0.623 & 3.836 & 2 \\
3 & 2.974 & 4.340 & 0.685 & 4.340 & 2 \\
4 & 0.088 & 0.116 & 0.758 & 0.116 & 2 \\
5 & 1.748 & 2.346 & 0.745 & 2.346 & 2 \\
6 & 0.082 & 0.105 & 0.780 & 0.105 & 2 \\
7 & 0.086 & 0.106 & 0.811 & 0.106 & 2 \\
8 & 0.091 & 0.139 & 0.654 & 0.139 & 2 \\
\hline
\end{tabular}

experiments 3 and 7, only K-means fails to get true clusters. In experiments 4 and 6, while GCC and K-medoids successfully obtain true clusters, Kmeans and $\mathrm{K}$-medians fails.

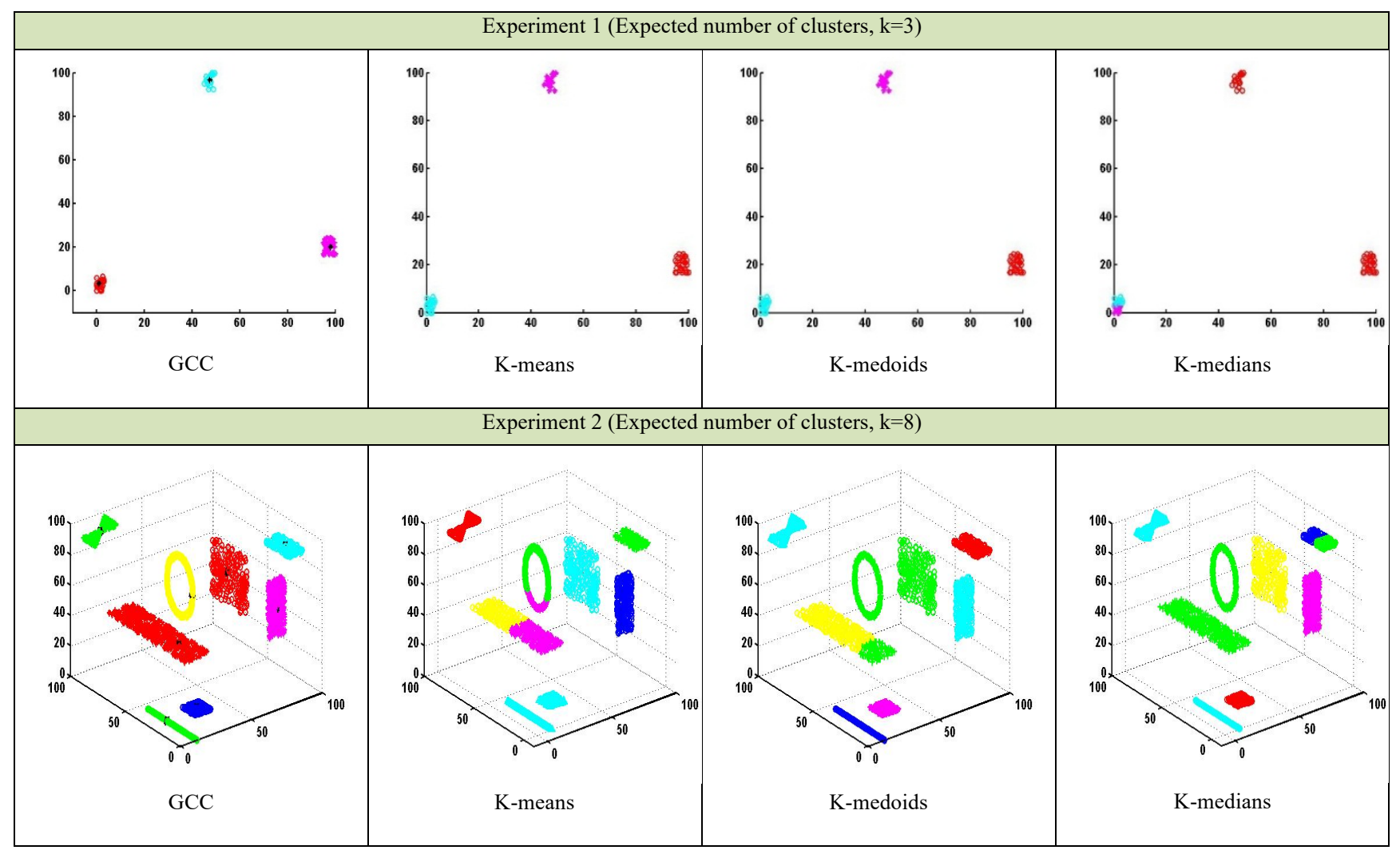




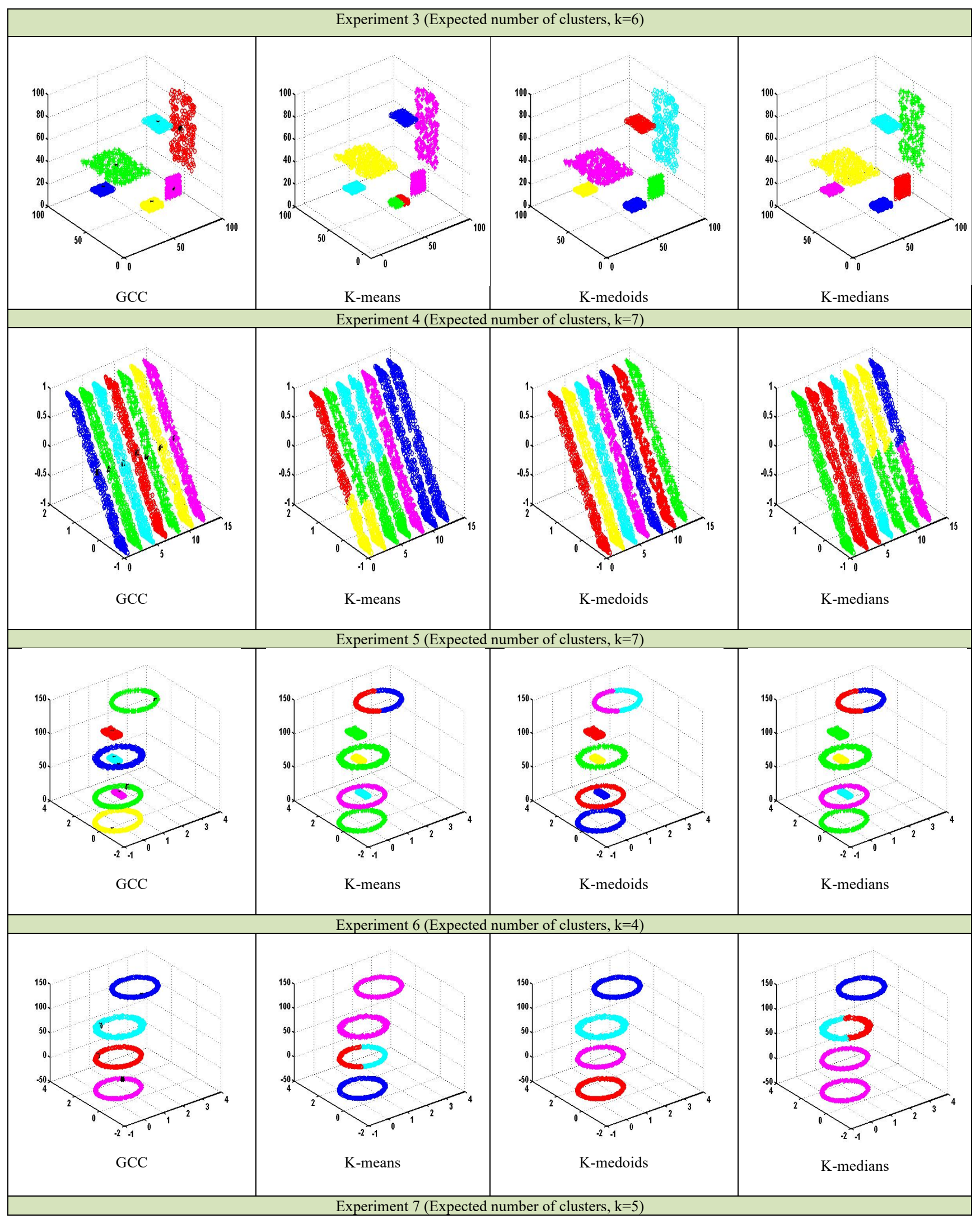




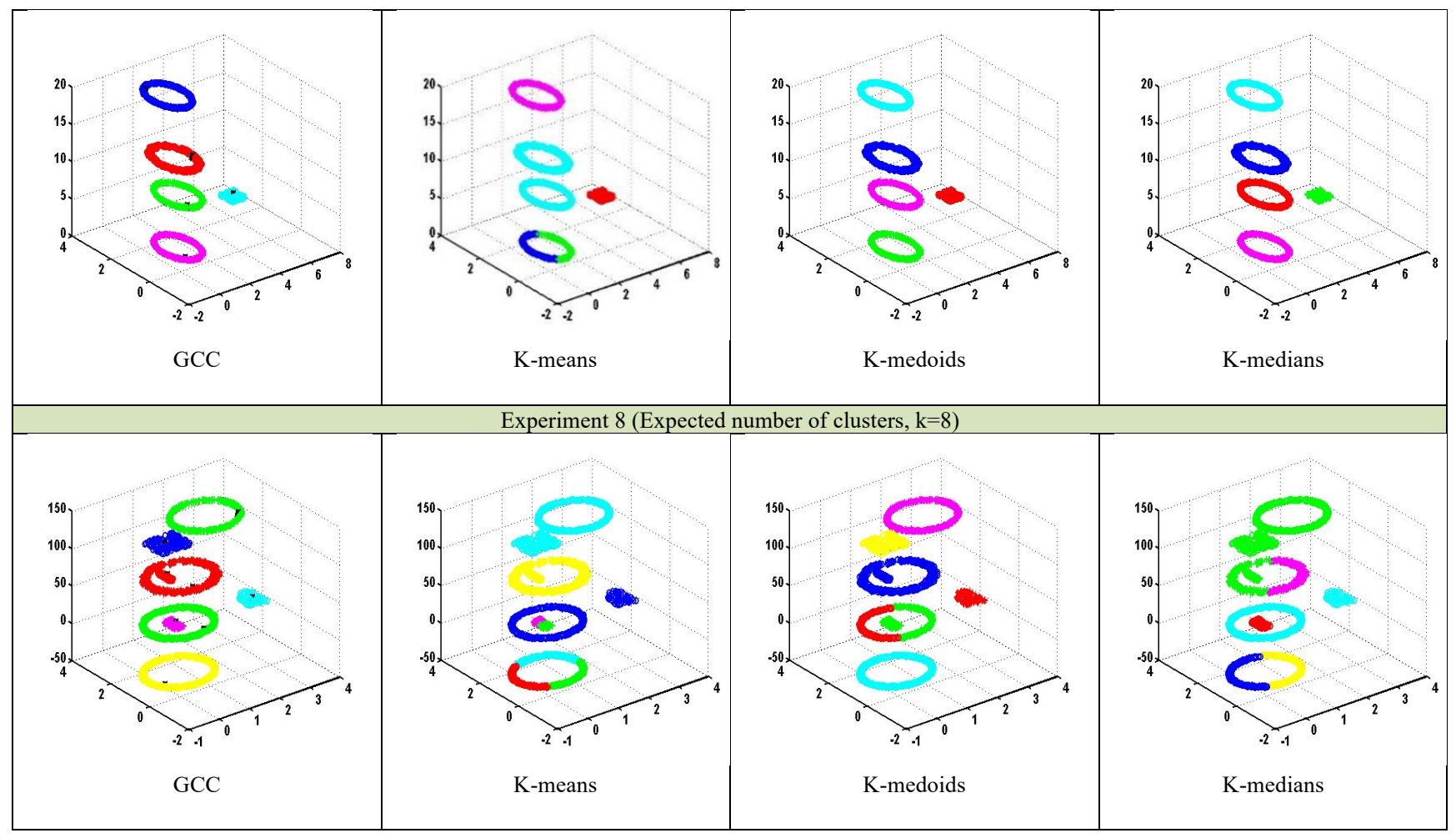

Fig. 2. The results of the first group of experiments.

4.2. Experiments group 2: Exploring the effect of coefficient $n$ using synthetic data

\begin{tabular}{ccccccc}
\hline $\begin{array}{c}\text { Exp. } \\
\text { No. }\end{array}$ & ULO & $\lambda$ & $\sigma$ & Threshold & $\mathrm{n}$ & $\begin{array}{c}\text { No. of } \\
\text { clusters }\end{array}$ \\
\hline $\mathbf{9}$ & $\mathbf{1 . 1 0 2}$ & $\mathbf{1 . 8 6 6}$ & $\mathbf{0 . 5 9 0}$ & $\mathbf{1 . 8 6 6}$ & $\mathbf{2}$ & $\mathbf{7}$ \\
& & & & & 7 & 6 \\
& & & & & 90 & 5 \\
$\mathbf{1 0}$ & $\mathbf{2 . 0 8 5}$ & $\mathbf{3 . 0 2 8}$ & $\mathbf{0 . 6 8 8}$ & $\mathbf{3 . 0 2 8}$ & $\mathbf{2}$ & $\mathbf{1 1}$ \\
& & & & & 7 & 10 \\
& & & & & 9 & 9 \\
$\mathbf{1 1}$ & $\mathbf{1 . 0 0 7}$ & $\mathbf{1 . 9 6 8}$ & $\mathbf{0 . 5 1 1}$ & $\mathbf{1 . 9 6 8}$ & $\mathbf{2}$ & $\mathbf{7}$ \\
& & & & & 360 & 3 \\
$\mathbf{1 2}$ & $\mathbf{2 . 3 0 1}$ & $\mathbf{3 . 6 1 3}$ & $\mathbf{0 . 3 5 3}$ & $\mathbf{3 . 6 1 3}$ & $\mathbf{2}$ & $\mathbf{8}$ \\
& & & & & 4 & 7 \\
& & & & & 19 & 6 \\
$\mathbf{1 3}$ & $\mathbf{2 . 0 5 7}$ & $\mathbf{4 . 0 1 5}$ & $\mathbf{0 . 5 1 2}$ & $\mathbf{4 . 0 1 6}$ & $\mathbf{2}$ & $\mathbf{7}$ \\
& & & & & 346 & 6 \\
& & & & & 702 & 3 \\
$\mathbf{1 4}$ & $\mathbf{3}$ & $\mathbf{1 . 2 1 6}$ & $\mathbf{2 . 3 2 5}$ & $\mathbf{1 . 2 1 6}$ & $\mathbf{2}$ & $\mathbf{6}$ \\
& & & & & 104 & 5 \\
& & & & & 321 & 3 \\
\hline
\end{tabular}

The second group experiments is also compatible with clustering. Thus, $\lambda$ is itself used as a first coefficient because $\sigma$ is not close to zero. Fig. 3 shows that outliers are not extreme, or noise cannot prevent getting clusters. Table 3 shows the coefficients and number of obtained clusters in each experiment where $\lambda$ used as threshold in all of them. Since the value of $n$ is optional and depending on user's desire, $\mathrm{n}$ is changed for all experiments to reduce the number of clusters according to the quorum criterion. For instance, in experiment 9 three results are obtained: 7 clusters when $n=2$, 6 clusters when $\mathrm{n}=7$ and finally 5 clusters when $\mathrm{n}=90$. In experiment 11 , 4 clusters contained the same number of points (360 points), hence all of

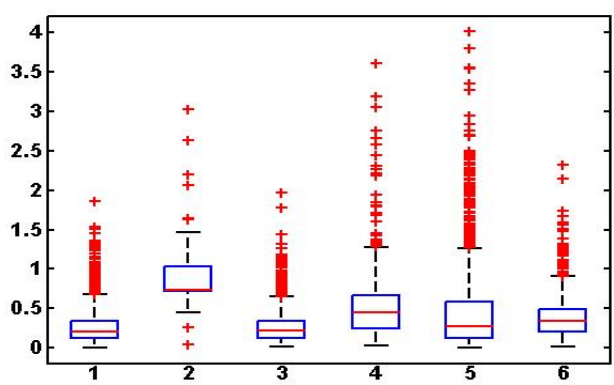

them are combined to their nearest clusters. Each cluster contained a point in black color representing the GC, therefore if the group does not contain

Fig. 3. ONE-WAY ANOVA graph for P2PS_M of the first group

that point it means it does not represent a cluster as it is merged with its closest cluster.

\section{Table 3.}

The coefficients of the second group of experiments.

The results of the second group are shown in Fig. 4, where the first column displays the GCC algorithm results with the default value of n, and the second and third columns represent the results of the GCC algorithm 
with several changes in $n$, except experiment 11 where only one change is made in $\mathrm{n}$.

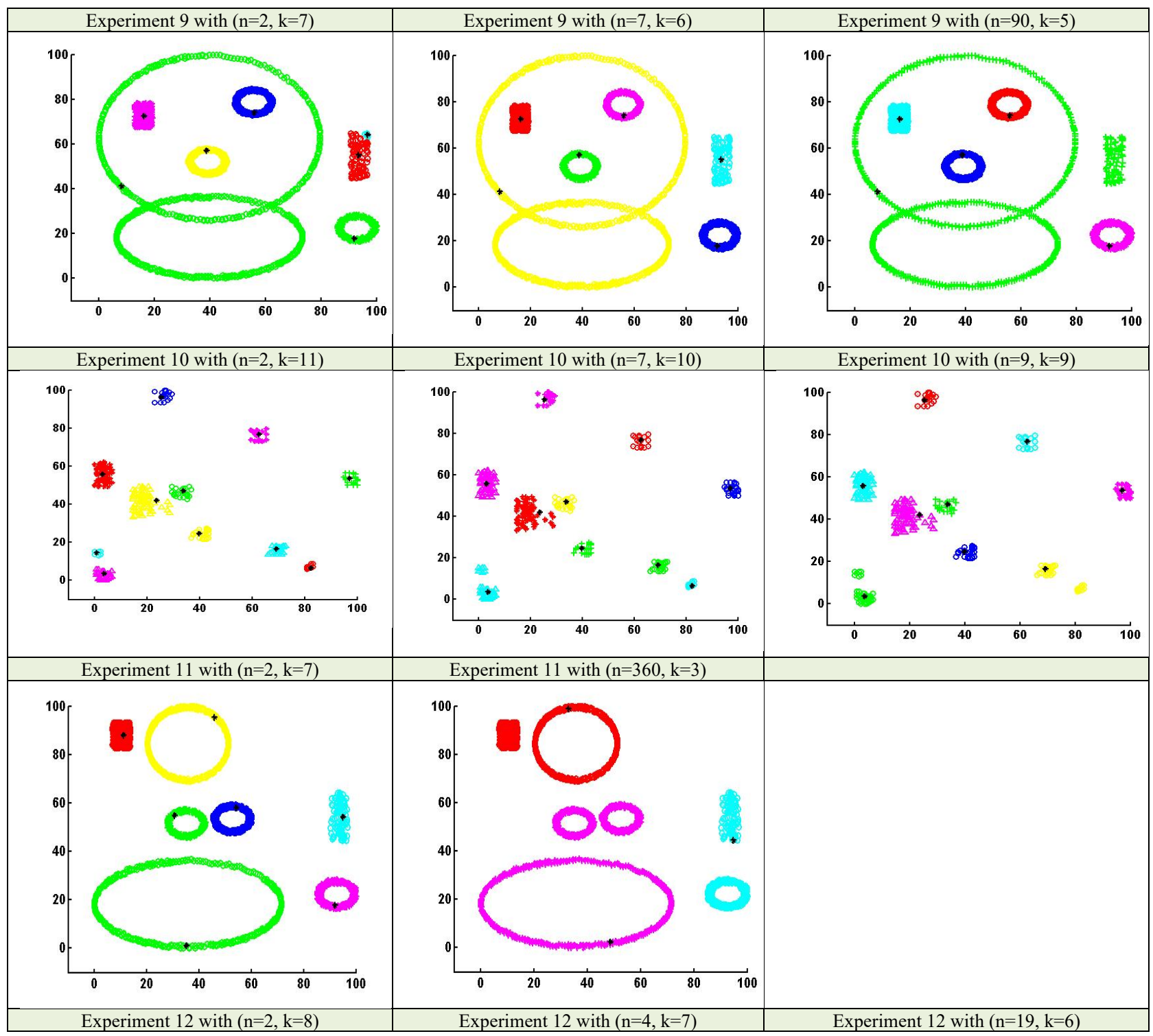




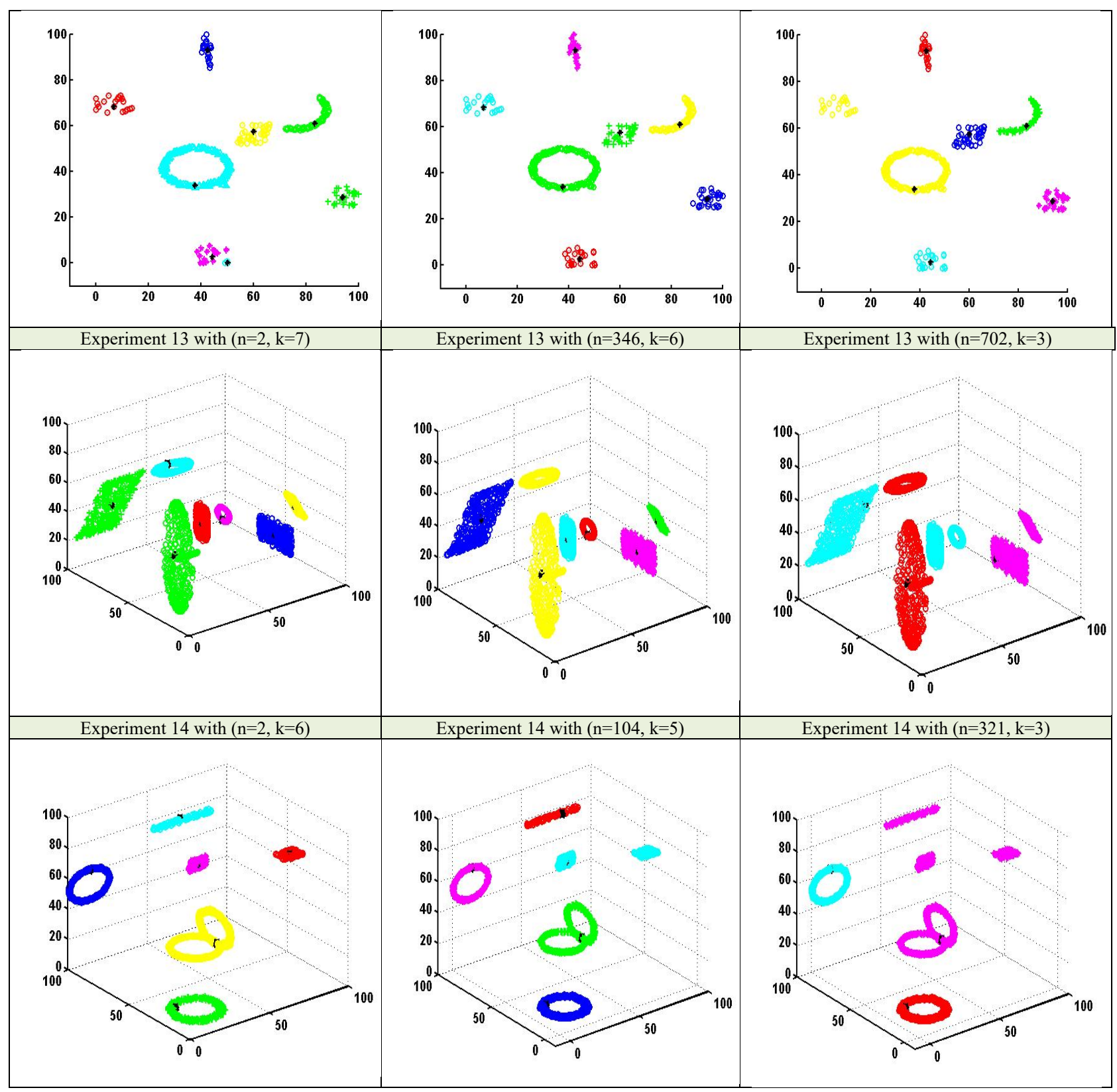

Fig. 4. The results of the second group of experiments.

4.3. Experiments group 3: Comparing GCC and K-means with changes in both coefficients using real data

Table 4 describes the features for 8 real healthcare datasets (DHIS, 2015a, 2015b, 2017, 2018; ODC-ODbL, 2016; CMS, 2017; UCI, 2016; CMS, 2015). As seen in Table 4, while experiments from 1 to 6 use 3 features of specified datasets, experiment 7 uses 2 features and experiment 8 uses 8 features. Due to the difficulty of perceiving more than 3 dimensions, clustering results for datasets with up to 3 features are presented.

Table 4.

Descriptions of datasets for the third group of experiments.

\begin{tabular}{|l|l|c|}
\hline \multicolumn{2}{|l|}{$\begin{array}{l}\text { Experiment 1 nndss-table-ii.-Varicella-to-West- } \\
\text { Nile-virus-disease }\end{array}$} & $\begin{array}{c}\text { Selected } \\
\text { features }\end{array}$ \\
\hline Feature 1 & Varicella (chickenpox), Cum 2013 & $\mathrm{F}$ \\
\hline Feature 2 & $\begin{array}{l}\text { West Nile virus disease, Neuroinvasive, } \\
\text { Previous 52 weeks Med }\end{array}$ & $\mathrm{H}$ \\
\hline Feature 3 & $\begin{array}{l}\text { West Nile virus disease, Neuroinvasive, } \\
\text { Previous 52 weeks Max }\end{array}$ & $\mathrm{L}$ \\
\hline $\begin{array}{l}\text { Experiment 2 nndss-table-ii.-salmonellosis-to- } \\
\text { shigellosis }\end{array}$ & $\begin{array}{c}\text { Selected } \\
\text { features }\end{array}$ \\
\hline Feature 1 & Salmonellosis, Current week & $\mathrm{D}$ \\
\hline Feature 2 & Salmonellosis, Previous 52 weeks Med & $\mathrm{F}$ \\
\hline
\end{tabular}




\begin{tabular}{|c|c|c|}
\hline Feature 3 & Salmonellosis, Previous 52 weeks Max & $\mathrm{H}$ \\
\hline \multicolumn{2}{|c|}{$\begin{array}{l}\text { Experiment } 3 \text { nndss-table-ii.-salmonellosis- } \\
\text { excluding-typhoid-fever-and-paratyphoid-fever-to- } \\
\text { shigellosis }\end{array}$} & $\begin{array}{l}\text { Selected } \\
\text { features }\end{array}$ \\
\hline Feature 1 & $\begin{array}{l}\text { Shiga toxin-producing Escherichia coli, } \\
\text { Current week }\end{array}$ & $\mathrm{N}$ \\
\hline Feature 2 & $\begin{array}{l}\text { Shiga toxin-producing Escherichia coli, } \\
\text { Previous } 52 \text { weeks Med }\end{array}$ & $P$ \\
\hline Feature 3 & $\begin{array}{l}\text { Shiga toxin-producing Escherichia coli, } \\
\text { Previous } 52 \text { weeks Max }\end{array}$ & $\mathrm{R}$ \\
\hline \multicolumn{2}{|c|}{$\begin{array}{l}\text { Experiment } 4 \text { nndss-table-ii.-babesiosis-to- } \\
\text { campylobacteriosis }\end{array}$} & $\begin{array}{l}\text { Selected } \\
\text { features }\end{array}$ \\
\hline Feature 1 & Babesiosis, Previous 52 weeks Max & $\mathrm{H}$ \\
\hline Feature 2 & Babesiosis, Cum 2016 & $\mathrm{~J}$ \\
\hline Feature 3 & Campylobacteriosis, Current week & $\mathrm{N}$ \\
\hline \multicolumn{2}{|c|}{ Experiment 5 health-infectious-disease-2001-2014 } & $\begin{array}{l}\text { Selected } \\
\text { features }\end{array}$ \\
\hline Feature 1 & Population & $\mathrm{F}$ \\
\hline Feature 2 & Rate & G \\
\hline Feature 3 & CI.upper & $\mathrm{F}$ \\
\hline \multicolumn{2}{|c|}{ Experiment 6 Unplanned Hospital Visits - Hospital } & $\begin{array}{l}\text { Selected } \\
\text { features }\end{array}$ \\
\hline Feature 1 & Score & $\mathrm{M}$ \\
\hline Feature 2 & Lower Estimate & $\mathrm{N}$ \\
\hline Feature 3 & Higher Estimate & $\mathrm{O}$ \\
\hline \multicolumn{2}{|c|}{ Experiment 7 dataset_diabetes } & $\begin{array}{l}\text { Selected } \\
\text { features }\end{array}$ \\
\hline Feature 1 & Blood Pressure & $\mathrm{C}$ \\
\hline Feature 2 & SkinThickness & $\mathrm{D}$ \\
\hline \multicolumn{2}{|c|}{$\begin{array}{l}\text { Experiment } 8 \text { Medicare National DMEPOS HCPCS } \\
\text { Aggregate CY } 2013\end{array}$} & $\begin{array}{l}\text { Selected } \\
\text { features }\end{array}$ \\
\hline Feature 1 & Number of Referring Providers & $\mathrm{F}$ \\
\hline Feature 2 & Number of Suppliers & G \\
\hline Feature 3 & Number of Supplier Beneficiaries & $\mathrm{H}$ \\
\hline Feature 4 & Number of Supplier Claims & $\mathrm{I}$ \\
\hline Feature 5 & Number of Supplier Services & $\mathrm{J}$ \\
\hline Feature 6 & Average Supplier Submitted Charges & $\mathrm{K}$ \\
\hline Feature 7 & Average Supplier Medicare Allowed & $\mathrm{L}$ \\
\hline Feature 8 & Average Supplier Medicare Payment & $\mathrm{M}$ \\
\hline
\end{tabular}

In this group of experiments, it is clear that the real data often does not accept the concept of clustering partially or completely because of the random distribution which causes the existence of many challenges such as outliers, overlapping and the existence of different regions in density and homogeneity of similarity. This exposes the ability and flexibility of the proposed algorithm to deal with such cases through the coefficients $\lambda$ and $\mathrm{n}$. Additionally, by identifying the ULO value, it is possible to determine whether the $\lambda$ is suitable for the threshold of each cluster. Fig. 5 represents the ONE-WAY ANOVA graph and Fig. 6 shows obtained clusters for the third group of experiments in which most of the experiments include outlier or overlapping. In the first experiment, existence of two outliers makes GCC assign all the points in one cluster. Therefore, GCC obtains 3 clusters either by excluding these points or by selecting an appropriate $\lambda$ by multiplying ULO. Since $n=2$, neither of both points can be an independent cluster, thus, it is merged with the nearest one.

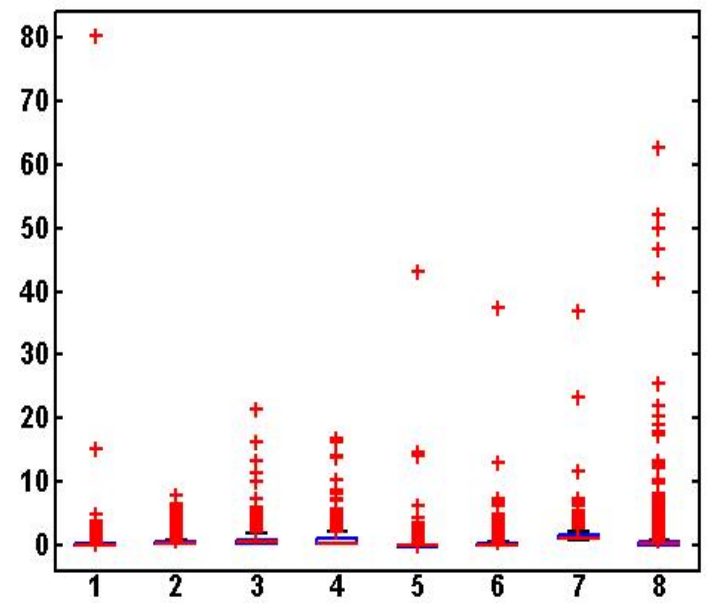

Fig. 5. ONE-WAY ANOVA graph for P2PS_M of third group

In Table 5, it is shown that the values of $\sigma$ in experiments 2, 3 and 4 are not close to zero, hence the value of $\lambda$ is suitable, whereas in experiment 1 we observe $\sigma$ value close to zero, thus ULO $* 7$ is used as an alternative to $\lambda$. The same applies to other experiments from 5 to 8 . Experiment 3 shows how to control the number of clusters by $\lambda$ (which is based on the similarity criterion to deal with merging operation). Whenever $\lambda$ value increases, it increases the number of points inside the cluster, thus, in turn, it leads to a reduction in the number of clusters by merging the clusters close to each other or those which have more similarity. While in experiment 5 where coefficient $\mathrm{n}$ is used, (which is based on the quorum criterion to merge the clusters), the weak clusters, where the number of points is less than $\mathrm{n}$ are included within nearest cluster.

It is important to point out here that the alternative $\lambda$ can be used from within the range known as threshold range. The threshold range extends from $\lambda$ value to distance required to contact the two closest clusters to each other, where $\lambda$ can be chosen in this range without affecting the results as shown in Table 5. For example, in experiment 1 the threshold is found between range ULO*7 and ULO*18.

Table 5.

The coefficients of the third group of experiments. 


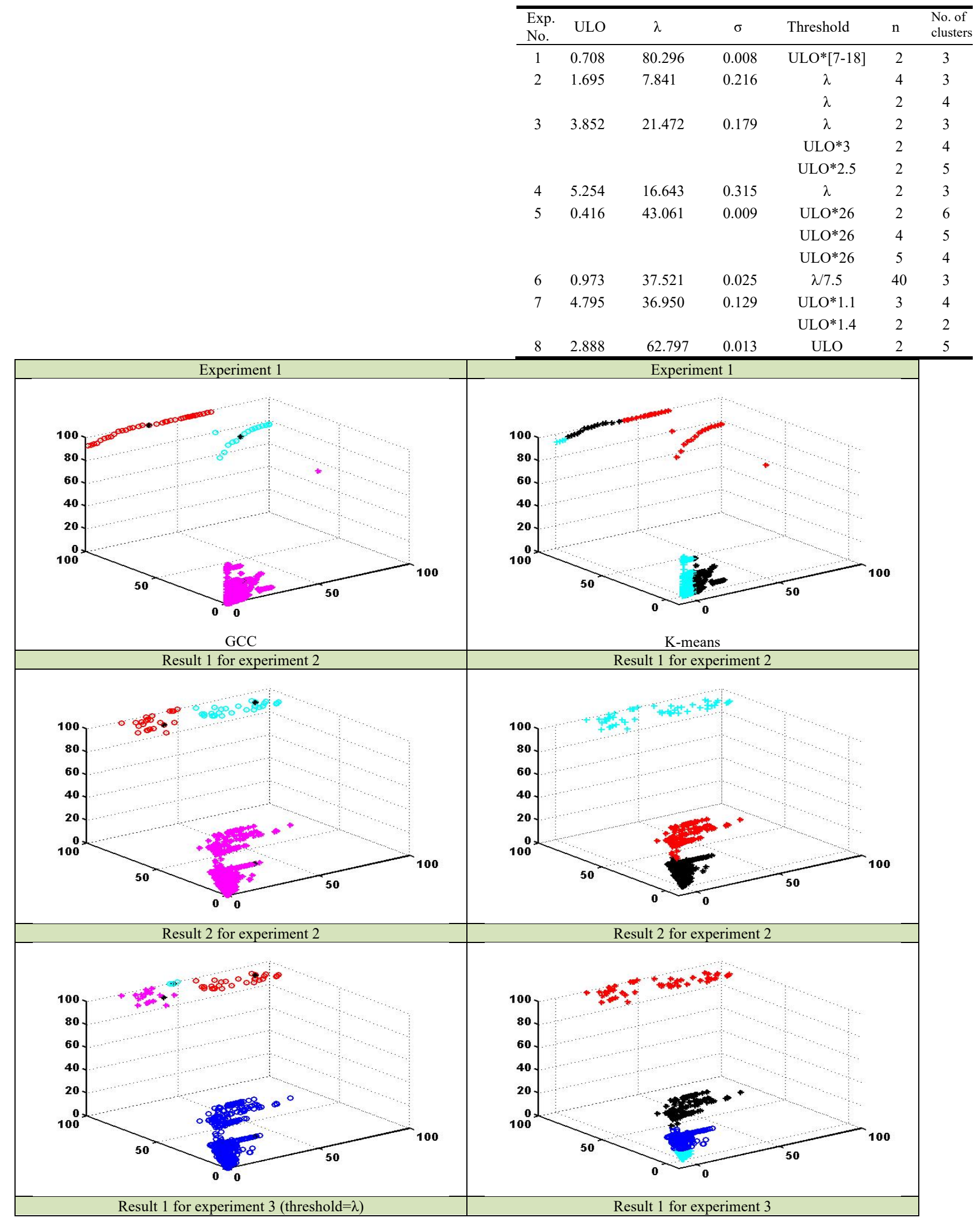




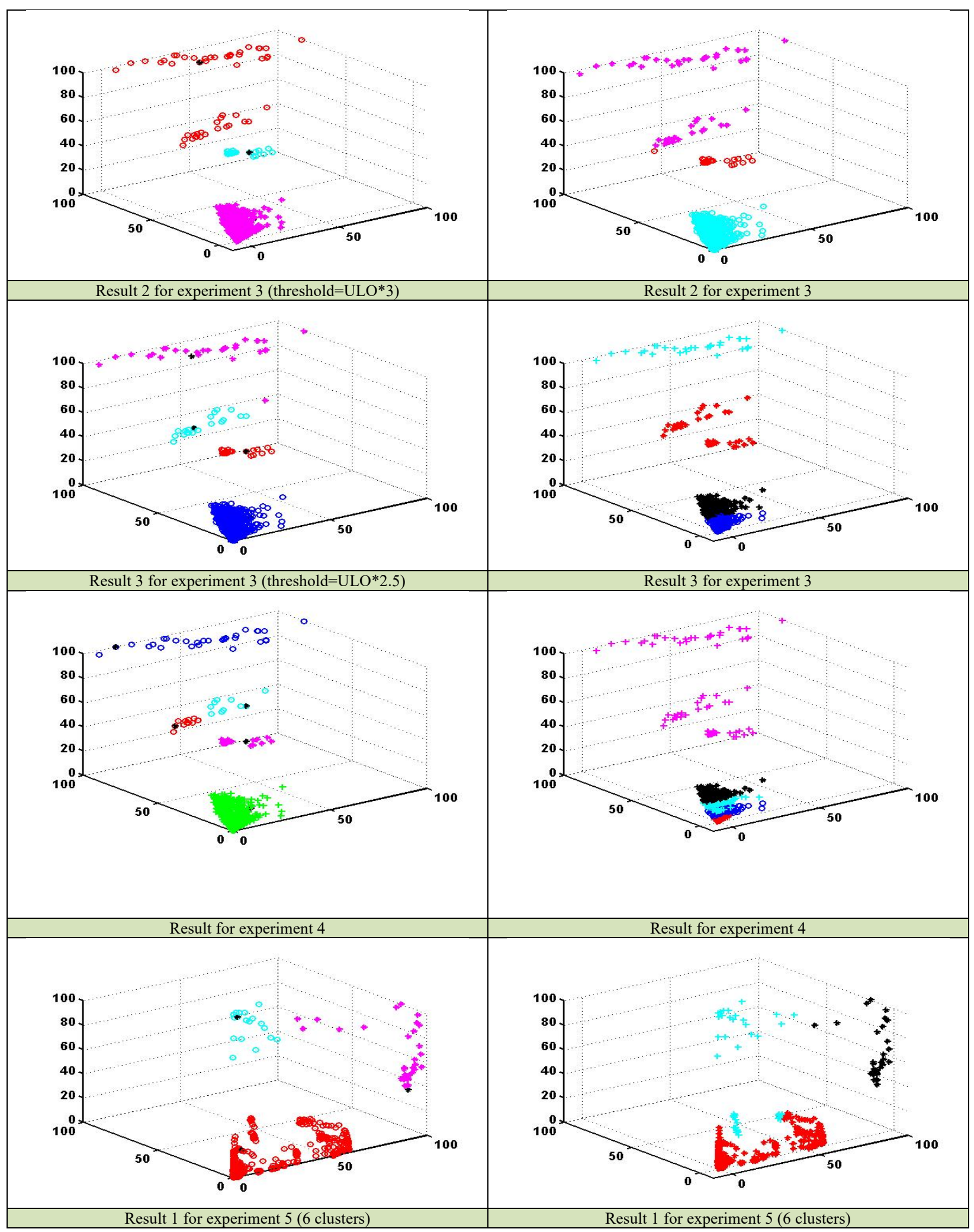




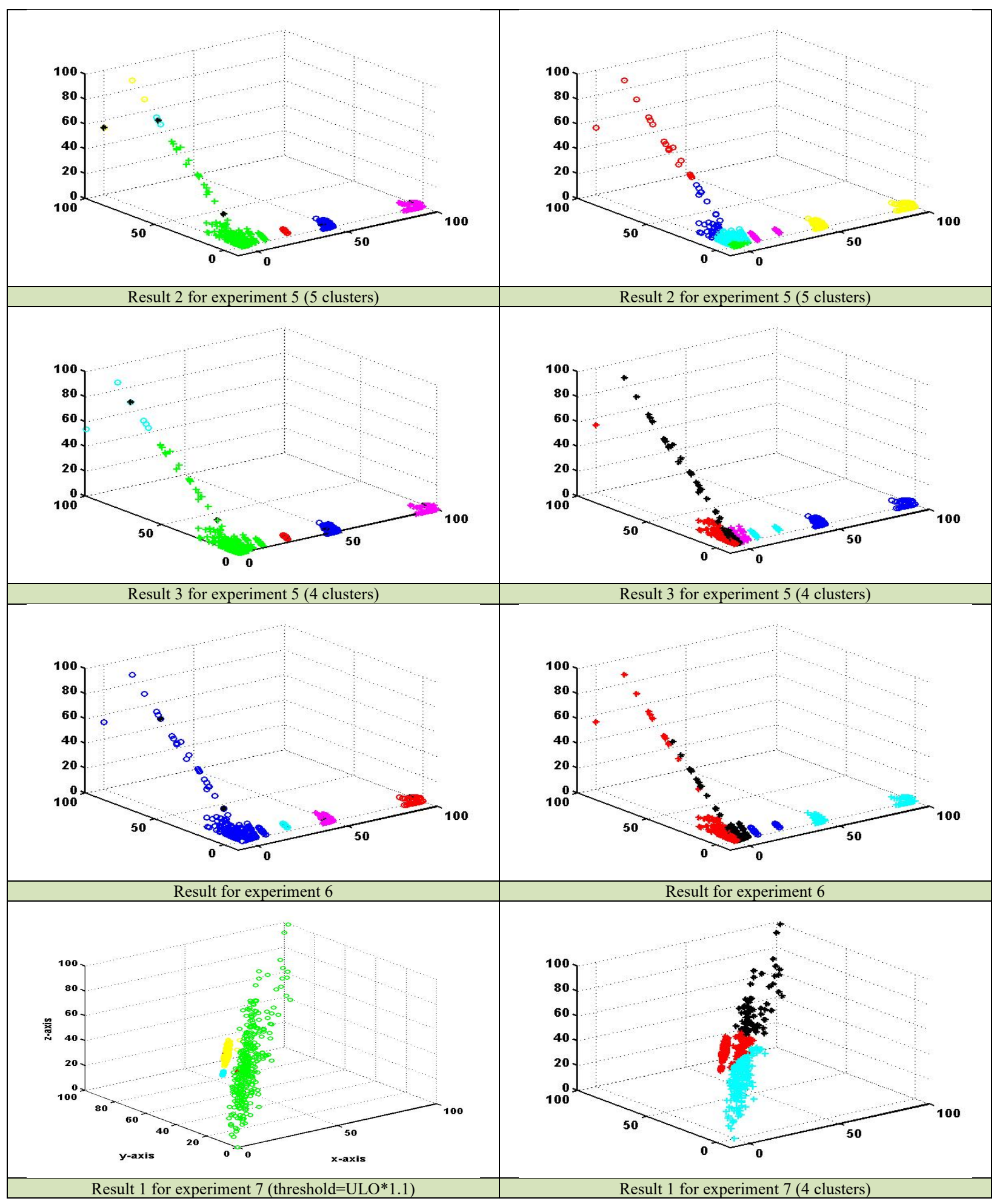




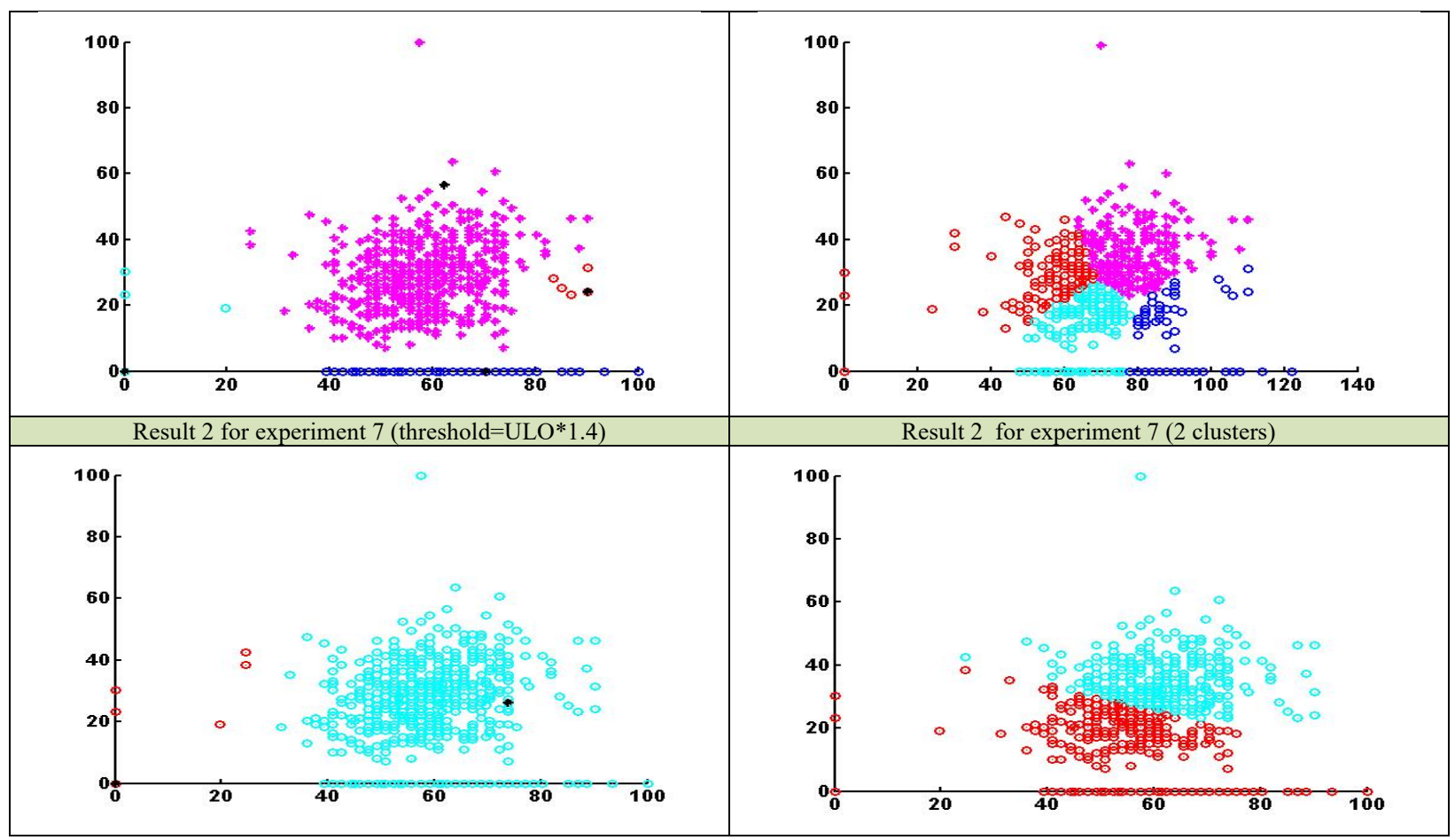

Fig. 6. The results of the third group of experiments.

\subsection{Speed analysis}

The performance of GCC is compared with other hard partitional algorithms in terms of execution time based on first group of experiments. The number of points in the data sets used in the experiments varies between 94 and 2520. Execution times of the algorithms and clustering results as true/false are given in Table 6 . While true clustering means the algorithm completely separates the clusters, false means one or more clusters are merged by the algorithm thus clustering is not true. In the table, $\mathrm{n}$ denotes number of points and $\mathrm{k}$ denotes the number of clusters in the dataset.

Table 6 also gives success rate of algorithms which is the ratio of the number of experiments performed with correct clustering to the total number of experiments. Due to the complexity of GCC that is $\mathrm{O}\left(\mathrm{n}^{2}\right)$, the algorithm performs clustering much slower than K-means, K-medians and K-medoids. As seen in the Table 6, while GCC algorithm gives best clustering performance with $100 \%$ success, it stands behind other algorithms in terms of execution speed. While GCC achieves high clustering quality, its cost to GCC is low execution speed in return. Kmedoids takes second highest time because it partially considers cohesion in addition to connectivity. On the other hand, K-means and K-medians have lowest runtimes. Both algorithms have similar times as well as similar clustering qualities. As general, much consideration of cohesion for an algorithm returns as more execution time.

Table 6.

Comparison of GCC with other hard partitional clustering algorithms according to execution time and success rate.

\begin{tabular}{ccccccccccc}
\hline & & & \multicolumn{2}{c}{ K-means } & \multicolumn{2}{c}{ K-medoids } & \multicolumn{2}{c}{ K-medians } & \multicolumn{2}{c}{ GCC } \\
\cline { 4 - 10 } Exp no & $\mathrm{n}$ & $\mathrm{k}$ & $\begin{array}{c}\text { Time } \\
(\mathrm{sec})\end{array}$ & $\begin{array}{c}\text { Clustering } \\
\text { result }\end{array}$ & $\begin{array}{c}\text { Time } \\
(\mathrm{sec})\end{array}$ & $\begin{array}{c}\text { Clustering } \\
\text { result }\end{array}$ & $\begin{array}{c}\text { Time } \\
(\mathrm{sec})\end{array}$ & $\begin{array}{c}\text { Clustering } \\
\text { result }\end{array}$ & $\begin{array}{c}\text { Time } \\
(\mathrm{sec})\end{array}$ & $\begin{array}{c}\text { Clustering } \\
\text { result }\end{array}$ \\
\hline 1 & 94 & 3 & 0.29 & True & 0.41 & True & 0.28 & False & 0.07 & True \\
2 & 2321 & 8 & 2.03 & False & 3.60 & False & 1.85 & False & 35.12 & True \\
3 & 1587 & 6 & 0.35 & False & 1.32 & True & 0.32 & True & 11.66 & True \\
4 & 2520 & 7 & 0.40 & False & 2.90 & True & 0.32 & False & 46.89 & True \\
5 & 2076 & 7 & 0.39 & False & 3.16 & False & 0.34 & False & 27.08 & True \\
\hline
\end{tabular}


F. H. Kuwil, U. Atila, R. Abu-Issa, and F. Murtagh/ Expert Systems with Applications

\begin{tabular}{ccccccccccc}
\hline 6 & 1440 & 4 & 0.32 & False & 0.94 & True & 0.30 & False & 9.49 & True \\
7 & 1544 & 5 & 0.33 & False & 0.97 & True & 0.31 & True & 11.28 & True \\
8 & 1825 & 8 & 0.36 & False & 2.32 & False & 0.31 & False & 16.79 & True \\
& Success rate & & $12.5 \%$ & & $62.5 \%$ & & $25 \%$ & & $100 \%$ \\
\hline
\end{tabular}

\section{Conclusions and future work}

In this paper, a novel partitional clustering algorithm GCC is proposed based on gravity center and a thresholding mechanism with two coefficients which provides the flexibility and ability to deal with challenges such as outliers, noise and overlapping. Results of GCC are compared to other wellknown hard partitional type clustering algorithms such as K-means, Kmedoids and $\mathrm{K}$-medians. Three groups of experiments were conducted with different synthetic and real datasets. First group of experiments showed that GCC was superior to other algorithms in obtaining true clusters. According to the results obtained in Experiment 1, GCC algorithm performed successful clustering in all eight experiments, whereas $\mathrm{K}$-means achieved correct clustering in 1 experiment, K-medians in 2 experiments and $\mathrm{K}$ medoids in 5 experiments. It should be noted that, datasets used in the first group of experiments were optimal and completely compatible with the concept of clustering meaning that there was no challenging case related to the distribution of data. Thus, conditions in the first group of experiments were suitable to get $100 \%$ true clustering for the algorithms, however, none of the implemented algorithms except GCC were successful in all experiments. Results of second group of experiments showed us how GCC can control clustering result by merging small clusters or clusters that does not reach required cluster size. Since extracting any pattern from such small clusters is not easy, it is a good advantage for user to be able to control this case. In third group of experiments, we showed how GCC can deal with challenging cases such as outlier and overlapping in real datasets.

Strengthens of our proposed GCC algorithm are as follows: a) Clustering quality of GCC comes into prominence over other algorithms. Since similarity of points in a dataset is based on connectivity and cohesion, unlike other algorithms, both principles are considered in GCC algorithm. Therefore, GCC produces more reasonable clusters than other algorithms regardless of data shape even though the dataset contains convex or concave shapes. Since, K-means and K-median completely ignore cohesion their results are worse than GCC and K-medoids. On the other hand, Kmedoids partially takes cohesion into account and this makes algorithm produce better results than K-means and K-medians but worse than GCC b) User does not need to specify any parameter in advance to implement GCC. Moreover, GCC provides two coefficients $\lambda$ and $n$ and an indicator sigma $\sigma$ which can be used for dealing with challenging situations such as presence of outliers, overlapping and weak clusters while other algorithms need other techniques or methods to be implemented along with c) Since clustering performance of GCC does not depend on initial random cluster centers unlike other algorithms, the results are robust and do not change from one run of the algorithm to other d) GCC works sequentially that, it extracts the most distinctive clusters first, and this puts GCC forward to overcome non-receptive data distribution problems while K-means, Kmedoids and K-medians work in parallel extracting all the clusters at the same time and this is one of the reasons that prevents these algorithms deal with distribution problems in data e) Since GCC algorithm depends on gravity center to discover the clusters, center point represents the core of the cluster which carries dominant features or characteristics of the cluster. Thus, the proposed clustering algorithm can be used prior to build a supervised learning model to help designing more efficient intelligent and expert systems.

Weakness of our proposed GCC algorithm is its computational complexity which is $\mathrm{O}\left(\mathrm{n}^{2}\right)$ in worst case. Especially when working with big datasets, time required for GCC is a critical limitation. This prevents GCC be a good alternative on large datasets for situations where cluster quality is compromised.

As feature work, weakness of GCC can be addressed by improving the runtime. Since GCC performs many calculations on matrices, it would be useful to build and analyze these matrices in incremental way in order to decrease complexity of the algorithm. To this aim, we work on a model that handles matrices in smaller parts.

\section{Acknowledgements}

We would like to express our gratitude to the management of the Department of Computer Engineering at Karabuk University for supporting our research by providing the use of Big Data laboratory. Also special thanks to Hamed Atia and Ali Belal for their support.

\section{References}

Agrawal, R., Gehrke, J., Gunopulos, D., Raghavan, P., Agrawal, R., Gehrke, J., ... Raghavan, P. (1998). Automatic subspace clustering of high dimensional data for data mining applications. $A C M$ SIGMOD Record, 27(2), 94-105.

Ahmadyfard, A., \& Modares, H. (2008). Combining PSO and k-means to 
enhance data clustering. In 2008 International Symposium on Telecommunications (pp. 688-691). IEEE.

Al-Sultan, K. S. (1995). A Tabu search approach to the clustering problem. Pattern Recognition, 28(9), 1443-1451.

Bezdek, J. C. (1981). Pattern Recognition with Fuzzy Objective Function Algorithms. Boston, MA: Springer US.

Blei, D. M., Ng, A. Y., \& Jordan, M. I. (2003). Latent Dirichlet Allocation. Journal of Machine Learning Research, 3(Jan), 9931022 .

Celebi, M. E., Kingravi, H. A., \& Vela, P. A. (2013). A comparative study of efficient initialization methods for the k-means clustering algorithm. Expert Systems with Applications, 40(1), 200-210.

Cheng-Fa Tsai, Han-Chang Wu, \& Chun-Wei Tsai. (2002). A new data clustering approach for data mining in large databases. In Proceedings International Symposium on Parallel Architectures, Algorithms and Networks. I-SPAN'02 (pp. 315-320). IEEE Comput. Soc.

Danyang Cao, \& Bingru Yang. (2010). An improved k-medoids clustering algorithm. In 2010 The 2nd International Conference on Computer and Automation Engineering (ICCAE) (pp. 132-135). IEEE. https://doi.org/10.1109/ICCAE.2010.5452085

Das, S., Abraham, A., \& Konar, A. (2008). Automatic kernel clustering with a Multi-Elitist Particle Swarm Optimization Algorithm. Pattern Recognition Letters, 29(5), 688-699.

Day, W. H. E., \& Edelsbrunner, H. (1984). Efficient algorithms for agglomerative hierarchical clustering methods. Journal of Classification, 1(1), 7-24.

DHIS. (2015a). NNDSS - Table II. Salmonellosis to Shigellosi. Retrieved from https://data.cdc.gov/NNDSS/NNDSS-Table-II-Varicella-toWest-Nile-virus-diseas/ig4m-ub43

DHIS. (2015b). NNDSS - Table II. Varicella to West Nile virus disease. Retrieved from https://data.cdc.gov/NNDSS/NNDSS-Table-IISalmonellosis-to-Shigellosis/52cr-rw4k

DHIS. (2017). NNDSS - Table II. Babesiosis to Campylobacteriosis. Retrieved from https://data.cdc.gov/NNDSS/NNDSS-Table-IIBabesiosis-to-Campylobacteriosis/4y34-2pku

DHIS. (2018). NNDSS - Table II. Salmonellosis (excluding typhoid fever and paratyphoid fever) to Shigellosis. Retrieved from https://data.cdc.gov/NNDSS/NNDSS-Table-II-Salmonellosisexcluding-typhoid-fev/6rpz-c2y5

Duda, R. O., Hart, P. E. (Peter E., \& Stork, D. G. (2001). Pattern classification (2nd Ed.). New York: Wiley.

Dunn, J. C. (1973). A Fuzzy Relative of the ISODATA Process and Its Use in Detecting Compact Well-Separated Clusters. Journal of Cybernetics, 3(3), 32-57.

Elfarra, B. K., EL Khateeb, T. J., \& Ashour, W. M. (2013). BH-centroids: A New Efficient Clustering Algorithm. BH-Centroids: A New Efficient Clustering Algorithm, Volume: 1,(Volume: 1, Number: $1)$.

Eschrich, S., Jingwei Ke, J., Hall, L. O., \& Goldgof, D. B. (2003). Fast accurate fuzzy clustering through data reduction. IEEE Transactions on Fuzzy Systems, 11(2), 262-270.

Ester, M., Kriegel, H.-P., Sander, J., \& Xu, X. (1996). A density-based algorithm for discovering clusters a density-based algorithm for discovering clusters in large spatial databases with noise. Proceedings of the Second International Conference on Knowledge Discovery and Data Mining. AAAI Press.

Figueiredo, M. A. T., \& Jain, A. K. (2002). Unsupervised learning of finite mixture models. IEEE Transactions on Pattern Analysis and Machine Intelligence, 24(3), 381-396.

Frigui, H., \& Krishnapuram, R. (1999). A robust competitive clustering algorithm with applications in computer vision. IEEE Transactions on Pattern Analysis and Machine Intelligence, 21(5), $450-465$.

Gil-Garcia, R. J., Badia-Contelles, J. M., \& Pons-Porrata, A. (2006). A General Framework for Agglomerative Hierarchical Clustering Algorithms. In 18th International Conference on Pattern Recognition (ICPR'06) (pp. 569-572). IEEE.

Guha, S., Rastogi, R., \& Shim, K. (1998). CURE:an efficient clustering algorithm for large databases. In Proceedings of the 1998 ACM SIGMOD international conference on Management of data SIGMOD '98 (Vol. 27, pp. 73-84). New York, New York, USA: ACM Press.

Halim, Z., \& Uzma. (2018). Optimizing the minimum spanning tree-based extracted clusters using evolution strategy. Cluster Computing, 21(1), 377-391.

Hamerly, G., \& Elkan, C. (2003). Learning the k in k -means. Proceedings of the 16th International Conference on Neural Information Processing Systems. MIT Press.

He, J., Zhao, G., Zhang, H. L., Ramamohanarao, K., \& Pang, C. (2014). An Effective Clustering Algorithm for Auto-Detecting WellSeparated Clusters. In 2014 IEEE International Conference on Data Mining Workshop (pp. 867-874). IEEE. 
Jain, A. K. (2010). Data clustering: 50 years beyond K-means. Pattern Recognition Letters, 31(8), 651-666.

Jain, A. K., Murty, M. N., \& Flynn, P. J. (1999). Data clustering: a review. ACM Computing Surveys, 31(3), 264-323.

Karaboga, D., \& Ozturk, C. (2011). A novel clustering approach: Artificial Bee Colony (ABC) algorithm. Applied Soft Computing, 11(1), 652-657.

Kauffmann L. (1987). Clustering by Means of medoids. Proc. Statistical Data Analysis Based on the L1 Norm Conference, Neuchatel, 1987, 405-416. Retrieved from https://ci.nii.ac.jp/naid/10027761751/

Kaufman, L., \& Rousseeuw, P. J. (1990). Finding groups in data : an introduction to cluster analysis. Wiley-Interscience.

Kuwil, F. H., Shaar, F., Topcu, A. E., \& Murtagh, F. (2019). A new data clustering algorithm based on critical distance methodology. Expert Systems with Applications, 129, 296-310.

Li, W., \& McCallum, A. (2006). Pachinko allocation. In Proceedings of the 23rd international conference on Machine learning - ICML '06 (pp. 577-584). New York, New York, USA: ACM Press.

McLachlan, G. J., \& Basford, K. E. (1988). Mixture models. Inference and applications to clustering. Statistics: Textbooks and Monographs, New York: Dekker, 1988.

Murtagh, F., \& Contreras, P. (2016). Linear Storage and Potentially Constant Time Hierarchical Clustering Using the Baire Metric and Random Spanning Paths (pp. 43-52). Springer, Cham.

Nanda, S. J., \& Panda, G. (2014). A survey on nature inspired metaheuristic algorithms for partitional clustering. Swarm and Evolutionary Computation, 16, 1-18.

Niknam, T., \& Amiri, B. (2010). An efficient hybrid approach based on PSO, ACO and k-means for cluster analysis. Applied Soft Computing, 10(1), 183-197.

Pelleg, D., \& Moore, A. W. (2000). Proceedings of the Seventeenth International Conference on Machine Learning (ICML-2000), June 29-July 2, 2000, Stanford University. Proceedings of the Seventeenth International Conference on Machine Learning. Morgan Kaufmann Publishers.

Saida, I. B., Kamel, N., \& Omar, B. (2014). A New Hybrid Algorithm for Document Clustering Based on Cuckoo Search and K-means (pp. 59-68). Springer, Cham.

Shelokar, P. ., Jayaraman, V. ., \& Kulkarni, B. . (2004). An ant colony approach for clustering. Analytica Chimica Acta, 509(2), 187-195.
Singh, A., Yadav, A., \& Rana, A. (2013). K-means with Three different Distance Metrics. International Journal of Computer Applications, 67(10), 13-17.

Verma, M., Verma, M., Srivastava, M., Chack, N., Diswar, A. K., \& Gupta, N. (2012). A Comparative Study of Various Clustering Algorithms. In Data Mining, International Journal of Engineering Research and Applications (IJERA), 1379-1384.

Wang, J. H., \& Rau, J. D. (2007). VQ-Agglomeration: a novel approach to clustering. IEE Proceedings - Vision, Image and Signal Processing (Vol. 148). Institution of Engineering and Technology.

Xu, R., \& Wunsch, D. (2005). Survey of Clustering Algorithms. IEEE Transactions on Neural Networks, 16(3), 645-678.

Zadeh, M. R. D., Fathian, M., \& Gholamian, M. R. (2014). A new method for clustering based on development of Imperialist Competitive Algorithm. China Communications, 11(12), 54-61.

Zhang, J., \& Shen, L. (2014). An Improved Fuzzy c -Means Clustering Algorithm Based on Shadowed Sets and PSO. Computational Intelligence and Neuroscience, 2014, 1-10.

Zhou, D., Cheng, Z., Wang, C., Zhou, H., Wang, W., \& Shi, B. (2004). SUDEPHIC: Self-Tuning Density-Based Partitioning and Hierarchical Clustering (pp. 554-567). Springer, Berlin, Heidelberg.

Zhou, R., Shu, L., \& Su, Y. (2015). An adaptive minimum spanning tree test for detecting irregularly-shaped spatial clusters. Computational Statistics \& Data Analysis, 89, 134-146. 